\title{
COMPLETENESS OF SINGLET PAIR STATES FOR QUANTUM SPIN SYSTEMS
}

\author{
W.J. CASPERS \\ Center for Theoretical Physics, University of Twente, P.O. Box 217, 7500 AE Enschede, The Netherlands
}

Received 8 July 1989; accepted for publication 7 September 1989

Communicated by A.A. Maradudin

\begin{abstract}
It is shown that for an arbitrary subdivision of an antiferromagnetic spin-1 lattice into two subsystems every singlet state can be expressed as a linear combination of single pair states (SPS). These SPS states are products of singlet states for a pair of representatives of either subsystem.
\end{abstract}

\section{Introduction}

Recently Oguchi and Kitatani [1] posed the question of the completeness of the singlet pair states (SPS) in relation with the construction of the ground state of an antiferromagnetic (AF) Heisenberg system $\left(S=\frac{1}{2}\right)$. This question is particularly interesting for the relevance of the so-called resonating-valencebond (RVB) approximation of the ground state, in which approximation the ground state is represented by a linear combination of SPS with proper phases. Anderson [2] originally introduced the method for the frustrated triangular lattice and Oguchi, Nishimori and Taguchi [3] used it for actual calculation of the ground-state energy of this system. Iske and Caspers [4] also applied it to nonfrustrated lattices and laid stress on the relation with the phenomenon of symmetry breaking. Liang, Doucot and Anderson [5] clearly demonstrated that the introduction of single pairs for nonneighbours is relevant for this symmetry breaking.

The aim of this paper is to give a simple proof of the (over)completeness of the set of SPS, with the further restriction that every singlet pair in an SPS is a combination of two spins of different sublattices. In the next section this proof will be given and in the final part of this paper a distinction will be made between bound and unbound pairs, which may be relevant for the analysis of the properties of Anderson's spinons, which play a role in his model for high- $T_{\mathrm{c}}$ superconductivity [6].

\section{Completeness of the set of single pair states}

The (over)completeness of the set of SPS for pairs of spins of different subsystems is a property independent of the choice of the subdivision of the spin system into these two subsystems with an equal number of spins, as follows from the proof given in this section. Then the most natural way of making this subdivision is into two sublattices, at least in the case of nonfrustrated systems, like the Heisenberg systems for the linear chain, the square and the cubic lattice. The well-known Hamiltonian for these systems has the form:

$H=J \sum_{\langle i, j\rangle} S_{i} \cdot S_{j}(J>0)$.

It is a sum of terms corresponding with neighbour pairs, the members of which pertain to the two sublattices. The Heisenberg systems defined by this Hamiltonian (1) are examples of systems for which the following theorem holds:

Theorem: For a system of $2 N$ spins $\frac{1}{2}$ subdivided into 2 subsystems of $N$ spins an arbitrary singlet state 1 > may be written as a linear combination of products of singlet states for pairs of the form:

$$
\left|1, j_{1}\right\rangle\left|2, j_{2}\right\rangle\left|3, j_{3}\right\rangle \ldots\left|N, j_{N}\right\rangle \text {. }
$$


The indices $1,2, \ldots, N$ refer to the spins of one subsystem, say $\mathbf{I}$, in a given order, whereas $j_{1}, j_{2}, j_{3}, \ldots$, $j_{N}$ indicate a permutation of the spins of the other subsystem II. The symbols $\left|n, j_{n}\right\rangle$ denote a singlet state for the pair $\left(n, j_{n}\right)$. There exist $N$ ! of these single states $(2)$ for the total system.

In the definition of the singlet pairs in (2) we introduce a definite phase convention given by

$$
\left|i_{1}, j_{1}\right\rangle=\frac{1}{\sqrt{2}}(|+,-\rangle-|-,+\rangle)_{\left(i_{1}, j_{1}\right)} \text {. }
$$

The first entry in the brackets in both members always refers to a spin of subsystem $I$, the second to one of subsystem II. The symbols + and - denote eigenstates of the $z$-components of both spins with eigenvalues $\pm \frac{1}{2} \hbar$.

Proof. The arbitrary singlet state | $>$ may always be developed in a series of the following form:

$$
|\rangle=\sum_{S M \beta \delta} b_{S M \beta \delta}|S, M ; \beta\rangle_{1, \mathrm{II}} \Psi_{\mathrm{I}^{\prime}}(S,-M ; \delta) \text {. }
$$

The numerical 1 indicates the first spin of the set I and I' denotes the complement of 1 in this set. The total spin $S$ of both sets $(1$, II) and I' are coupled to total spin zero. The spin $S$ may have the values 0,1 , $2, \ldots(N-1) / 2$ for odd $N$, and $\frac{1}{2}, \frac{3}{2}, \ldots,(N-1) / 2$ for even $N$. The second quantum number $M$ or $-M$ corresponds with the $z$-component of the total spin of the two sets and the symbols $\beta, \delta$ denote additional quantum numbers. It is implied that the reader is acquainted with the general theory of angular momentum in quantum mechanics (cf. ref. [7]), in particular with the addition of two momenta.

In the state $|S, M ; \beta\rangle_{1, \mathrm{II}}$ one may distinguish 2 parts:

$$
|S, M ; \beta\rangle_{1 \mathrm{II}}=|S, M ; \beta\rangle_{1 \mathrm{II} ; \mathrm{1}}+|S, M ; \beta\rangle_{\mathrm{III} ; 0},
$$

in which the second part is a linear combination of terms that are antisymmetric in at least one pair (1, $j), j$ denoting a spin of set II. The first part is completely symmetric in all pairs $(1, j)$.

A crucial step in the argument is that this first part is identical to zero, because full symmetry in all pairs $(1, j)$ implies full symmetry in all pairs $\left(j, j^{\prime}\right)$ and consequently in the whole set $(1, \mathrm{II})$. This leads to the conclusion that $S$ should have the value $(N+$ 1) $/ 2$ but this value of the total spin cannot be re- alized for the set $\mathrm{I}^{\prime}$, so it cannot give a contribution to the sum in (4). Then it follows immediately that | > may be written as

|\rangle$=\sum_{j} c_{j}|1, j\rangle\left|\mathbf{I}^{\prime}, \mathbf{I I}_{j}\right\rangle$.

In this expression the right member is a sum of products of singlet states, the first corresponding with the pair $(1, j)$, the second with the complementary sets in I and II respectively. Now the argument leading to (6) may be repeated till finally a linear combination of expressions of the form (2) is reached and the statement of the theorem is proved.

The two subsystems I, II may be chosen to correspond to the sublattices of a nonfrustrated antiferromagnetic lattice, but for the argument leading to the statement of the theorem this is in no way relevant. It may help, however, to give the reader insight in the physical relevance of this theorem for the properties of antiferromagnetic systems described by the Hamiltonian (1).

Some remarks are in order as to the number of singlet states of the type (2). It is easily demonstrated that this number equals $N$ !, representing the number of permutations of the spins of one subsystem. The total number of SPS, without the restriction that in every pair there is a representative of two given subsystems, equals

$D_{2 N}=\frac{(2 N) !}{N ! 2^{N}}$,

according Oguchi and Kitatani [1] for a lattice of a total number of spins $2 \mathrm{~N}$. The total number of linearly independent singlets, however, may be found by taking the difference of the number of independent states with $M_{0}=0$ and $M_{0}=1$ and this number equals

$D_{0.2 N}=\frac{(2 N) !}{(N) !(N) !}-\frac{(2 N) !}{(N-1) !(N+1) !}=\frac{(2 N) !}{N !(N+1) !}$.

The symbol $M_{0}$ represents the $z$-component of the total spin.

The (over) complete set we consider in this paper has a number of elements between (7) and (8), as follows from a little algebra:

$D_{0,2 N} \leqslant N ! \leqslant D_{2 N}$. 
In table 1 we give values of these three numbers for small $N$.

In this paper we are only interested in the two columns of table 1 corresponding with $D_{0,2 N}$ and $N$ !. First we try to understand the difference between the two entries for $N=3$. In this case we have two groups of 3 spins, which may be indicated by $I(1,2,3)$ and II $(4,5,6)$.

The symbols I and II again refer to 2 subgroups (sublattices) with members $(1,2,3)$ and $(4,5,6)$. One may think of 6 spins on a ring in the order 1 , $4,2,5,3,6$, with neighbour pair interactions between spins of different groups. The coupling for each neighbour pair is of the type (1). The 6 singlet states of the column $N$ ! are represented by (2) with $N=3$ and $\left(j_{1}, j_{2}, j_{3}\right)$ being one of the permutations:

$$
\begin{aligned}
& (4,5,6),(4,6,5),(5,4,6), \\
& (5,6,4),(6,4,5),(6,5,4) .
\end{aligned}
$$

Simple algebra leads to the following relation between the 6 singlets:

$$
\begin{aligned}
& |1,4\rangle|2,5\rangle|3,6\rangle-|1,4\rangle|2,6\rangle|3,5\rangle \\
& \quad-|1,5\rangle|2,4\rangle|3,6\rangle+|1,5\rangle|2,6\rangle|3,4\rangle \\
& \quad+|1,6\rangle|2,4\rangle|3,5\rangle-|1,6\rangle|2,5\rangle|3,4\rangle=0,
\end{aligned}
$$

from which it follows that there cannot exist more than 5 independent singlet states, which is confirmed by the value of $D_{0,2 N}$ for this case. For the system of 8 spins with two subgroups of 4 spins there exist 24 singlets of the type (2). For each subset of 6 spins, with an equal number of spins of either sublattice a relation of the type $(10)$ holds, which relations together result in a number of 14 independent singlets. We have the strong conviction that the

Table 1

Number of singlet states of a system of $2 N$ spins.

\begin{tabular}{rrrr}
$N$ & $D_{0,2 N}$ & \multicolumn{1}{c}{$N !$} & \multicolumn{1}{c}{$D_{2 N}$} \\
\hline 1 & 1 & 1 & 1 \\
2 & 2 & 2 & 3 \\
3 & 5 & 6 & 15 \\
4 & 14 & 24 & 105 \\
5 & 42 & 120 & 945 \\
6 & 132 & 720 & 10395 \\
\hline
\end{tabular}

elimination of singlets of the type (2) in the case of an overcomplete set can always be realized by relations of the type (10) for a subset of 6 spins. In the next section we pay attention to the physical meaning of the SPS of the type (2) in relation with antiferromagnetic ordering in a Heisenberg system. A distinction will be made between bound and (asymptotically) free singlet pairs.

\section{Bound and unbound singlet pairs. Spinons}

Before discussing in detail the existence of bound and unbound pairs within the RVB scheme we want to visualize the idea of RVB a bit more. This will be done with the help of fig. 1 in which a picture of a special "switchboard" is drawn, which graphically represents one single SPS.

The two sublattices are respectively represented by a circle (I) and by a black dot (II). Connections can be made between points of I and II by a "cable" i.e. a vector, indicated by an arrow. The vectors have an energy, because they represent a singlet pair, e.g. the pair $\left(i_{1}, j_{1}\right)$, which in this example is a neighbour pair in a two-spin state. According to the Hamiltonian (1) such a pair has an energy $-\frac{3}{4} J$.

For all singlet pairs with a larger distance the energy is 0 , so there is a tendency for the members of a pair to occupy neighbouring sites, at least in a state of low energy for the total spin system. These spin pairs, however, are "resonating", i.e. the stationary states of (1), are linear combinations of states of the type (2). The Hamiltonian induces transitions between these states according to special selection rules, which may be illustrated by fig. 2 . The transition pictured in this figure is induced by the coupling be-

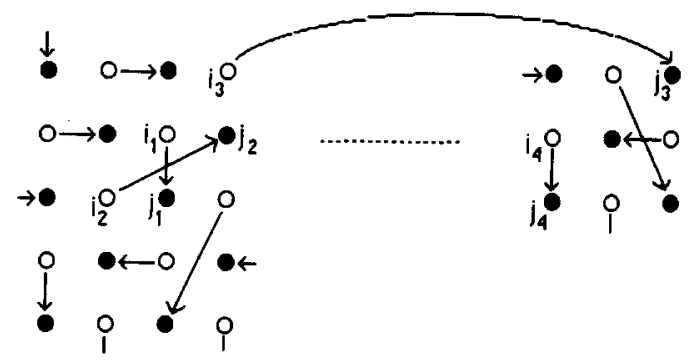

Fig. 1. Graphical representation of one single SPS. 


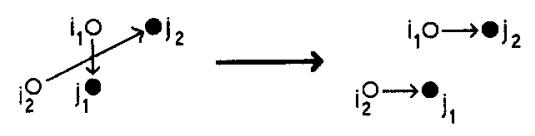

Fig. 2. Transition between 2 SPS configurations.

tween the neighbours $\left(i_{1}, j_{2}\right)$ (or by the pair $\left(i_{2}, j_{1}\right)$ ) corresponding with the term $J S_{i_{1}} \cdot S_{j_{2}}$ (or $J S_{i 2} \cdot S_{j_{1}}$ ).

The above statements about the (secular) energy and the transitions between SPS are formally expressed by the formulae:

$$
\begin{aligned}
& H_{i j_{1}}\left|i_{1}, j_{1}\right\rangle\left|i_{2}, j_{2}\right\rangle=-\frac{3}{4} J\left|i_{1}, j_{1}\right\rangle\left|i_{2}, j_{2}\right\rangle, \\
& H_{i 2 j_{1}}\left|i_{1}, j_{1}\right\rangle\left|i_{2}, j_{2}\right\rangle \\
& =+\frac{1}{4} J\left(\left|i_{1}, j_{1}\right\rangle\left|i_{2}, j_{2}\right\rangle-2\left|i_{1}, j_{2}\right\rangle\left|i_{2}, j_{1}\right\rangle\right) .
\end{aligned}
$$

These two formulae enable us, in principle, to solve the secular problem for the complete set of singlet states of Heisenberg systems. All these singlet states are linear combinations of those depicted in fig. 1, with the proviso that not all states are linearly independent.

In the ground state we expect that SPS with pairs on relatively short distances are predominant. This was recently confirmed by the paper of Liang et al. [5] already cited, in which it was also demonstrated that for large but finite spin systems the contribution of non-neighbour pairs is essential for the existence of long-range order.

Wave-like excitations (spin waves) of low energy may be represented by linear combinations of the SPS with phases and amplitudes that approach (quasi) continuously those of the ground state, in the limit of long waves. Apart from these well-known excitations we expect those of an other type, which behave more like solitons. This is illustrated by the pair $\left(i_{3}, j_{3}\right)$ in fig. 1 , which is supposed to have a large distance between its two constituents. For a finite system there is no qualitative difference between pairs on short and large distances. For (infinitely) large systems, however, one may distinguish bound and free pairs, the last category being represented by a linear combination of SPS in which a pair like $\left(i_{3}\right.$, $j_{3}$ ) appears with an amplitude that has a finite limit for a distance that approaches infinity. So far this is mere speculation but the two categories are wellknown in the literature, in the form of the already mentioned spin waves and in that of (pairs) of spinons in the Anderson theory of high- $T_{\mathrm{c}}$ supercon- ductivity. We expect that the spinons represent a nonvanishing energy, a property already discussed for the square lattice by Kivelson, Rokhsar and Sethna [8]. Maybe our picture may eliminate the existing controversy with regard to this finite energy (cf. ref. [6]).

In nonstationary states one may expect that two spinon wave packets may annihilate one another under the creation of ordinary spin waves.

A remark is in order as to the creation of spinons in the Anderson model of superconductivity. In essence this is a Hubbard model with an asymptotically large positive interaction between the fermions [9]. For near half filling of the lattice the holes in this system act as the charge carriers. For holes at a fixed positions in the lattice the fermion gas (electrons) behaves like an ideal Heisenberg antiferromagnet, in which excitations of the types discussed above may occur. The motion of holes, however, is coupled with the creation of spinons, which are expected to play a crucial role in the superconducting properties the model claims to describe. The relevance of the underlying paper may be found in better understanding of the concept of spinon.

\section{Acknowledgement}

The author acknowledges the stimulating discussions with Paul Iske and the technical support of this colleague in the preparation of this text.

\section{References}

[1] T. Oguchi and H. Kitatani, J. Phys. Soc. Japan 58 (1989) 1403.

[2] P.W. Anderson, Mater. Res. Bull. 8 (1973) 153.

[3] T. Oguchi, H. Nishimori and Y. Taguchi, J. Phys. Soc. Japan 55 (1986) 323.

[4] P.L. Iske and W.J. Caspers, Physica A 142 (1987) 360.

[5] S. Liang, B. Doucot and P.W. Anderson, Phys. Rev. Lett. 61 (1988) 365.

[6] P.W. Anderson, G. Baskaran, Z. Zou and T. Hsu, Phys. Rev. Lett. 58 (1987) 2790.

[7] A.R. Edmonds, Angular momentum in quantum mechanics, 2nd ed. (Princeton Univ. Pres, Princeton, 1980).

[8] S.A. Kivelson, D.S. Rokhsar and J.P. Sethna, Phys. Rev. B 35 (1987) 8865.

[9] J. Hubbard, Proc. R. Soc. A 276 (1963) 238; A 277 (1964) 237. 